\title{
Revista

\section{PERSPECTIVAS SOBRE EL BUEN VIVIR EN UN MUNICIPIO TSOTSIL DE LOS ALTOS DE CHIAPAS}

Paola Ortelli

Universidad Autónoma de Chiapas / Universidad Autónoma Metropolitana-I paorte@yahoo.com

Marcos Gómez López Universidad Autónoma de Chiapas / Ayuntamiento Municipal de San Andrés Larráinzar marcush_85@hotmail.com

\section{RESUMEN}

En este artículo se exponen los resultados de la investigación enmarcada en el proyecto «Cuestionando el desarrollo: hacia prácticas de buen vivir». En este sentido, a partir del caso del municipio tsotsil de San Andrés Larráinzar, se compararán los discursos de los jóvenes y de los mayores sobre el lekil kuxlejal (buen vivir). Se enfatiza sobre la perspectiva de los mayores, quienes afirman la importancia del sistema de cargos tradicional en la continuidad de la vida del pueblo, así como su función ético-educativa para los jóvenes originarios del municipio en cuestión. Se avanzan hipótesis explicativas sobre la asociación del buen vivir con el sistema de cargos, presentando resultados preliminares de una investigación doctoral en proceso.

PALABRAS CLAVE: buen vivir, sistema de cargos.

\section{PERSPECTIVES REGARDING GOOD LIVING IN A TSOTSIL MUNICIPALITY IN THE CHIAPAS HIGHLANDS}

\section{ABSTRACT}

This article presents results from a piece of research framed within a project entitled «Questioning Development: Towards Practices of Good Living». Based on the case of the tsotsil municipality of San Andrés Larráinzar, it compares the discourse of youth and the elderly regarding lekil kuxlejal (good living/buen vivir). It highlights the perspective of tsotsil elders, who reaffirm the importance of the traditional ladder of responsibilities (cargo system) for continuity of the people's lives, as well as the ethical-educational function the elders perform for the tsotsil youth in this municipality. Presenting preliminary results from a PhD piece of research in process, it sets forth explanatory hypotheses regarding the association between good living/buen vivir and the traditional ladder of responsibilities.

KEY WORDS: good living/buen vivir, cargo system. 


\section{INTRODUCCIÓN}

E ntre marzo de 2011 y agosto de 2012, como Cuerpo Académico «Etnia, estado y desarrollo» de la Universidad Autónoma de Chiapas, obtuvimos un financiamiento del Programa de mejoramiento del profesorado (PROMEP) para un proyecto de investigación titulado «Cuestionando el desarrollo: hacia prácticas de buen vivir».

Una de las estrategias metodológicas implementadas en el marco del proyecto fue la organización de cuatro foros en diferentes municipios de los Altos de Chiapas, mismos que tenían el objetivo general de confrontar opiniones y discursos de diferentes actores locales sobre el tema del buen vivir.' Queríamos explorar hasta qué punto los planteamientos boliviano y ecuatoriano podían ser pertinentes para la región de los Altos de Chiapas. Lo que analizaremos en este artículo serán los resultados del foro que se llevó a cabo en el municipio tsotsil de San Andrés Larráinzar, ${ }^{2}$ que fue el único caso en el que surgió una definición del buen vivir en la que sobresale la dimensión ético-política del concepto, planteado como vivir como pueblo, en el respeto del sistema de cargos. En este sentido analizaremos el concepto en la formulación boliviana y ecuatoriana y, sucesivamente, en la mexicana. Después expondremos los resultados del foro y, a partir de ellos, buscaremos profundizar sobre los factores y dinámicas que influyen en la formulación que autoridades y ex autoridades constitucionales del municipio de estudio hicieron del concepto, tratando de explicar ¿Por qué en este caso se habla del buen vivir como vivir en el respeto del sistema de cargos y qué implica esto en su significado?

\section{SOBRE EL CONCEPTO DE BUEN VIVIR}

El planteamiento del «buen vivir», se origina en los conceptos aymara y kitchwa de suma qamana ('vivir bien' o 'coexistir en armonía con la naturaleza y sus semejantes') y sumak kawsay 'buen vivir' o 'vida en plenitud', que el intelectual aymara Fernando Huanacuni Mamani define así: «Vivir bien, es la vida en plenitud. Saber vivir en armonía y equilibrio; en armonía con los ciclos de la Madre Tierra, del

\footnotetext{
${ }^{1}$ Los foros se llevaron a cabo en cuatro municipios de los Altos de Chiapas.

${ }^{2}$ Cabe mencionar que el foro se organizó con la parte constitucional del Ayuntamiento y lo que se presenta en este artículo de ninguna manera pretende agotar todas las perspectivas. Por supuesto que la historia específica del municipio es considerada como un elemento fundamental en el tipo de discurso que las autoridades manejan sobre el tema del buen vivir.
} 
cosmos, de la vida y de la historia y en el equilibrio con toda forma de existencia en permanente respeto» (2010:15). El buen vivir apunta a disociar la calidad de vida del crecimiento económico y de la destrucción del planeta. En este sentido este paradigma constituye una reacción al modelo de desarrollo convencional y corresponde asimismo a un modo de vida propicio a la preservación de una tierra viable para todos y cuyos recursos son repartidos de manera equitativa. Como señala Boaventura de Sousa Santos (2014) para el caso ecuatoriano el reconocimiento de la naturaleza como un ser vivo y por tanto limitado la convierte en sujeto y objeto de cuidado y de derechos y rompe a la vez con la idea de la apropiación ilimitada de los recursos naturales con la finalidad de saciar el consumismo y satisfacer un crecimiento económico sin límites. Así, a pesar de las diferencias y particularidades de los casos ecuatoriano y boliviano, ${ }^{3}$ en ambos países el paradigma del buen vivir se sustenta en la relación armoniosa con los demás y con la naturaleza alterando la ideología que asocia el desarrollo material a la idea de progreso. Se plantea así un doble desafío que implica la unión de los seres humanos entre ellos y con el medioambiente en el que viven. El buen vivir implica un buen convivir, basado en la unidad, el intercambio, la solidaridad, la reciprocidad y el respeto como valores fundamentales.

En México, uno de los primeros análisis sobre el concepto de buen vivir lo realiza Antonio Paoli, quien ha analizado el concepto tseltal de lekil kuxlejal 'buscar vivir bien' o 'buscar la buena vida', profundizando en los aspectos filosóficos que lo sustentan. Uno de los elementos que Paoli señala en el caso tseltal es la profunda integración entre la sociedad y la naturaleza. Asimismo «Hay lekil kuxlejal cuando existe slamalil kinal» (2003:71), es decir, paz interior y exterior, en el medio externo y en la mente, y solo se logra gracias a la existencia de una dimensión intercomunitaria e intersubjetiva que hace que «todos los participantes constituyen activos e influyentes transformadores de los otros en vista de un objetivo común» (2003:211). Finalmente, en cuanto filosofía de vida, el lekil kuxlejal «es la vida buena por antonomasia. No es una utopía porque no se refiere a un sueño inexistente. No, el lekil kuxlejal existió, se ha degradado pero no se ha extinguido y es posible recuperarlo» (2003:221). Constituye por lo tanto un horizonte al que aspirar como pueblo. Y es a partir de esta idea de horizonte común que encontramos un elemento de análisis interesante para el caso de estudio que es la materia de este artículo. Lo que nos interesa resaltar aquí es que, en el municipio

\footnotetext{
${ }^{3}$ Es importante mencionar que en ambos casos el paradigma del buen vivir se encuentra inscrito en la Constitución de Bolivia (2009) y de Ecuador (2008).
} 
estudiado, el concepto no se plantea en términos de lekil kuxlejal, sino más bien de kuxlejal 'buscar la vida', 'vivir', con énfasis en que el meollo del problema no es calificar las condiciones de vida, sino simplemente vivir como pueblo y para eso solo hay una forma posible que es la tradicional, antigua, la que se manifiesta en el respeto del sistema de cargos. En los párrafos siguientes presentaremos las percepciones que los habitantes de este municipio alteño tienen sobre el buen vivir buscando explicar los elementos que influyen en esta idea del sistema de cargos como el vehículo del buen vivir del pueblo.

\section{ALGUNOS ASPECTOS METODOLÓGICO-CONCEPTUALES}

Para conocer las perspectivas sobre la vida buena en el municipio en cuestión, gracias al apoyo del ayuntamiento constitucional y de dos profesionistas tsotsiles originarios del lugar, el 28 de octubre de 2011 se realizó un foro4 titulado Sloil slekilal kuxlejal xchi'uk mol me-eletik, xchi'uk ach' chi'eletik 'Diálogo sobre el buen vivir entre ancianos y jóvenes'. La idea consistía en generar un espacio de intercambio entre actores de diferentes generaciones con el fin de propiciar la reflexión, el diálogo, la concientización y posiblemente la construcción de propuestas encaminadas al buen vivir / lekil kuxlejal del municipio.

A nivel metodológico el foro se estructuró en dos momentos: una mesa redonda en la que se buscó conocer la perspectiva de los sabios sobre el kuxlejal y una reflexión de grupos de trabajo interétnicos e intergeneracionales conformados por los participantes del foro y facilitados por profesionistas originarios del municipio, orientada a ver sus perspectivas sobre el lekil kuxlejal y buscar elementos de continuidad o ruptura entre el planteamiento de los mayores y el de los demás actores. Las intervenciones fueron grabadas y traducidas simultáneamente por profesionistas andreseros bilingües, quienes facilitaron los trabajos de grupo. ${ }^{5}$ En total participaron 37 personas: dos autoridades municipales constitucionales (sexto y segundo regidor), tres alcaldes tradicionales (Primero, Segundo y Tercero), un pasaro (ex primer regidor), una partera, representantes de organiza-

${ }^{4}$ Al foro participaron 37 personas: autoridades municipales constitucionales, alcaldes, pasaros y exautoridades, representantes de organizaciones artesanas, jóvenes profesionistas del municipio y 11 estudiantes mestizos e indígenas procedentes de diferentes municipios de la Licenciatura en Gestión y Autodesarrollo Indígena de la Universidad Autónoma de Chiapas (UNACH), profesores de la misma universidad y de la Universidad Intercultural de Chiapas (UNICH).

${ }^{5}$ Véase Ortelli 2011 Relatoría del foro Sloil slekilal kuxlejal xchi'uk mol me-eletik, xchi'uk ach' chi'eletik. Traducción de Juana Pérez de la Torre. 
ciones artesanas, cinco profesionistas y jóvenes del municipio y 11 estudiantes mestizos e indígenas procedentes de diferentes municipios de la Licenciatura en Gestión y Autodesarrollo Indígena de la Universidad Autónoma de Chiapas, profesores de dicha universidad y de la Intercultural de Chiapas (UNICH).

Una de las hipótesis de partida del foro consistió precisamente en la idea que existe una diferencia de perspectiva entre los mayores y los jóvenes.

Ahora bien, antes de analizar los resultados del foro es necesario definir el concepto de jóvenes. En este texto coincidimos con Pérez (2008), quien asume la juventud como una categoría que se define culturalmente. Alpízar y Bernal (2003) hablan de esa etapa como un proceso de construcción social, con base en seis enfoques que, desde diferentes perspectivas, se disputan la definición del concepto.

Una perspectiva que ha tenido mucho impacto es la juventud como etapa problemática y de riesgo en el desarrollo psicobiológico. La corriente ha sido influenciada por el psicoanálisis, la psicología del desarrollo y los estudios sociológicos de corte funcionalista (Lidz 1973). Otro enfoque, desarrollado a mediados del siglo XX, concibe la juventud como momento clave para la integración social. Como «proceso de transición», constituye una etapa en la que la gente joven debe formarse y adquirir los valores que le permiten integrarse socialmente a una vida adulta (Erikson 1951). Desde esta perspectiva la juventud es un «estatus» que el individuo adquiere al adecuarse a ciertas actividades socialmente definidas. En forma simultánea se ha desarrollado la perspectiva de la juventud como dato sociodemográfico, definido a partir de la pertenencia a cierto grupo de edad definido arbitrariamente con el efecto homogeinizador. Influenciada por el materialismo histórico, se ha desarrollado la perspectiva de la juventud como agente de cambio. En este enfoque se idealiza a los jóvenes, identificándolos como principales agentes de cambio, contestatarios y anticonformistas, portadores de valores «posmaterialistas» (Inglehart 1990). Vinculada con el desarrollo de las políticas públicas, emerge la concepción de esta etapa como problema de desarrollo a causa del desempleo, el alto consumo de drogas ilícitas y el número de embarazos adolescentes (Ferraroti 1981). Preocupados por la integración social de los jóvenes (Turaine 1988), los autores que parten de este enfoque miran los problemas macroeconómicos e institucionales, aun cuando retoman las particularidades regionales y nacionales que viven los jóvenes (Rodríguez 1995a, 1995b). Finalmente, una última perspectiva apunta a considerar la juventud como grupo generacional identificado con ciertos acontecimientos históricos particulares de una determina- 
da época, homogeneizando a todas las personas ubicadas en una misma generación y reduciendo los conflictos a la división generacional (Coupland 1993). Como reacción a los enfoques antes expuestos, considerados «homogeneizantes», «estigmatizantes» y «adultocentristas», Alpízar y Bernal (2003) proponen la perspectiva de la construcción social de las juventudes, que encuentra sus antecedentes en las reflexiones desarrolladas por Park, Trasher y Mead (citados en Alpízar y Bernal 2003). Desde este enfoque la juventud está en un continuo proceso de construcción y reconstrucción histórica y «cada sociedad define 'la juventud' a partir de sus propios parámetros culturales, sociales, políticos y económicos, por lo que no hay una definición única» (Alpízar y Bernal 2003:18). Asimismo la construcción social de la juventud resalta la diversidad de formas de expresión de lo juvenil, tanto en términos de la existencia de múltiples culturas juveniles, como de una diversidad de lo juvenil como identidad (Feixa 1995).

Desde esta perspectiva hablar de jóvenes en Larráinzar implica alejarse del punto de vista meramente sociodemográfico, generacional, psicosocial e institucional, ya que el criterio de definición depende del tipo de responsabilidades que las personas adquieren respecto de la familia y la comunidad. En este texto nos referimos a los jóvenes como aquellos que «aún tienen que aprender a vivir», aún no han tomado cargos o apenas empiezan a aprender cómo servirle a la gente y a los santos. La definición, en el caso de estudio, incluye entonces a estudiantes de bachillerato y de la universidad, así como a mujeres y hombres sin experiencia en los cargos. Es importante observar que las mayores posibilidades de escolarización y profesionalización, así como la influencia del discurso del Estado sobre los derechos juveniles contribuyen a modificar patrones culturales, y de ese modo amplían el rango de edad que corresponde al concepto de jóvenes como categoría.

\section{SISTEMA DE CARGOS Y BUEN VIVIR: PERSPECTIVAS}

En el caso analizado se habló de kuxlejal 'vivir', más que de lekil kuxlejal 'vida buena', ya que se parte del supuesto de que la única manera de vivir es en el respeto de los cargos. Como mencionó una autoridad, "hay motivos, saberes, y razones, en cada uno de los cargos», con énfasis en su función ético-educativa, aunada a la preocupación de que los jóvenes que los asumen «no saben las razones de realizar un cargo porque no toman en cuenta las sabidurías que tiene uno 
con experiencia y es por eso que los cargos se van perdiendo». Se insistió sobre la importancia de seguir con los cargos, ya que:

[...] son los que encaminan a nuestro pueblo las enseñanzas que nos dejaron los ancianos, hay que obedecer lo que nos dicen, cada cargo tiene sus razones, la manera en que se le habla a nuestro padre y madre, cuando obedecemos siempre se nos queda algo sobre el vivir de los ancianos (Ortelli 2011).

Por otra parte la preocupación por su abandono por parte de los jóvenes introdujo un problema relevante, relacionado con el impacto de la educación superior en las regiones indígenas, así como con las expectativas de los mayores hacia la formación se sus jóvenes.

Como mencionó una autoridad:

[...] es bueno que el estudiante aprenda cosas como aprender a hablar español, pero también sería bueno que aprendieran a hablarle al pueblo tomando los cargos [...] Los jóvenes piensan que el vivir del pueblo es malo, no es bueno aprender y conocer estos cargos, existen muchachas y jóvenes que llegan a la iglesia y sí saben cómo hablarle a los Santos de la iglesia [...] por eso no hay que perder las costumbres y las tradiciones no hay que pensar que el vivir del pueblo es malo sino que es bueno (Ortelli 2011).

Estas palabras dejan entrever una ruptura entre las percepciones sobre el kuxlejal 'vivir' del pueblo de los mayores y de los jóvenes profesionistas y estudiantes, formados desde una perspectiva occidental y que aprenden a ver la cultura propia (Bonfil 1991) con una mirada valorativa y peyorativa.

En este sentido los cargos cumplen entonces una función formativa y ética, ya que enseñan los valores que encaminan al pueblo como entidad colectiva. En Larráinzar, como en el caso de los tseltales estudiados por Paoli, son las autoridades quienes, a través de su quehacer, deben educar a la dimensión intersubjetiva y comunitaria del lekil kuxlejal, ya que:

Se necesita también que ese con cargo respete al pueblo. No sólo cuando se sienta con autoridad para mandar, también cuando mete a la cárcel a quienes perturban a la gente. Se requiere que abra caminos al arreglo justo, que abra caminos al lekil kuxlejal. El lekil kuxlejal requiere que hagamos justicia con rectitud, y eso puede ser cuando no hay rencor en nuestro corazón hacia otro hermano y sentimos entonces armonía en el ambiente (Paoli 2003:82). 
El mensaje que los mayores quisieron transmitir a los jóvenes fue el del peligro implicado en la pérdida de dicha costumbre.

A esto se acompañó la recomendación de no perder el diálogo con los mayores, como única estrategia visualizada para mantener vivo el interés hacia las tradiciones del pueblo, amenazadas por el cambio de mentalidad debido, por un lado, a la experiencia escolar, pero también por la migración. Como mencionó un participante:

[...] les daría un ejemplo como son los jóvenes que se van para los Estados Unidos, ya no regresan normales, ya se integran en bandas y se ponen otros tipo de ropa, a su familia le habla en español, y eso es lo que destruye, porque ya no son los consejos que dejaron nuestros padres, y habría que cambiar eso, y cuando se viste bien veríamos que es alguien bueno, la imagen depende de cómo se vista una persona, cuando ya no nos importe casi nada eso no es bueno porque repercute a la sociedad (Ortelli 2011).

Es importante observar cómo se vincula la percepción negativa hacia la migración al problema de las bandas juveniles, a la introducción de valores diferentes que ponen en crisis los tradicionales y a la pérdida de un elemento cultural fundamental como la lengua, a través de la cual se transmite la forma de ser de una cultura.

En el planteamiento de los mayores, las tradiciones representan también aquel conjunto de valores cuyo seguimiento garantiza que el pueblo continúe por el camino del kuxlejal, unido, en armonía, hacia un mismo fin común. Se trata de una guía de carácter espiritual, que ayude a «no caer en otras religiones» que, a pesar de no ser «malas», se considera que «han creado problemas dentro de la comunidad», ya que de alguna manera impiden la posibilidad de seguir con un horizonte común como pueblo.

En las reflexiones grupales interétnicas e intergeneracionales los jóvenes rescataron los consejos de los mayores identificando una serie de valores que nos llevan a la vida buena, como la importancia del respeto al «otro» a través de actitudes no agresivas y la humildad. La reflexión, más allá de buscar definir el concepto de kuxlejal, se centró en los obstáculos para conseguirlo, entre los cuales se menciona el hecho de avergonzarse de las propias raíces. Como sostiene una joven originaria del municipio, uno de los pasos más importantes para lograrlo es por medio de la autovaloración y la valoración de la identidad propia: 
[...] nunca hay que avergonzarnos de nuestro origen y cultura, digamos que somos de un paraje somos de un origen, si me pongo mi traje tradicional no debo de avergonzarme. Un ejemplo que cuando comamos frijol tendríamos que prestar una cuchara para poder comer, cuando esté presente otra persona externa de la comunidad [...] y eso está mal, porque no nos deberíamos de avergonzar, ya que así vivimos, otra cosa es respetar nuestras vivencias, no nos debemos de avergonzar de nada hay que usar el traje tradicional, y la cultura de lo que hay que hacer, y cuando es de nuestra cultura y tradición (Ortelli 2011).

En el diálogo intergeneracional emerge el desconocimiento de los jóvenes sobre el sistema de cargos, cuya lógica es conocida únicamente por aquellas autoridades que están a punto de culminar una gestión (alcaldes, nakanhuanejes) y que se aprenden únicamente de manera vivencial al cumplir con el servicio. ${ }^{\circ}$ En este sentido emerge una manera diferente de conceptualizar el buen vivir entre mayores y jóvenes, quienes, aun cuando se apropian de los consejos de los mayores sobre el kuxlejal, introducen nuevos temas, como la equidad de género, la dimensión más individual del estar bien con uno mismo y la libertad de pensamiento y de credo, factores que, de acuerdo con los actores locales, amenazan la posibilidad de armonía del municipio y constituyen elementos de preocupación para los jóvenes. Como menciona un estudiante:

El buen vivir es estar bien, tener salud, alimentación, hogar, tener un trabajo, el lekil kuxlejal abarca muchas cosas, como estar bien en familia, el lekil kuxlejal es tener salud, y armonía familiar para luego estar bien en la sociedad y para tener una vida plena. Por otra parte el lekil kuxlejal tiene que ver con el sistema social, económico para que pueda satisfacer las necesidades como educación y alimentación, salud, estar en armonía en parte la naturaleza es un vínculo con quien estamos enlazados, porque es donde vivimos y respiramos a través de ello con los árboles. Cuidar a la naturaleza es cuidar una parte de nosotros porque sin la naturaleza no hay donde saliera los alimentos, los productos que se venden (Ortelli 2011).?

Sin embargo, es importante subrayar que ninguno de los jóvenes presentes en el foro ha expresado abiertamente un rechazo o una crítica al sistema de cargos, cuyo valor fundamental, fundado en el respeto del principio jerárquico de mayor

${ }^{6}$ Un análisis más profundo al respecto se desarrolla en uno de los capítulos de la tesis doctoral en proceso. Ortelli, capítulo 3 de la tesis.

${ }^{7}$ Intervención de un estudiante en el grupo 2. 
a menor, no es cuestionado y va más allá de las opiniones personales. Como menciona un joven:

Tenemos siempre que respetar, y preguntar es una forma de respeto. Yo todavía le pregunto a mis papas, para que no se pierda nada y así respetaríamos todo, y así no se perdería nada porque es ahí el comienzo de la tradición, respeto, que respetamos a nuestros padres y abuelos que los tomamos en cuenta, lo que ellos nos dicen por qué es bueno y aprenderíamos bien y también aprenderíamos a obedecer, a veces no lo creemos pero siempre hay que respetar, al igual a la familia, y hay que aprender lo que debe de ser como son los resultados de la obediencia y de los consejos, y solo está ahí, y solo tiene que tener una sola platica de donde fueron a aprender pero quien debe de aprender que es el mismo pueblo, y lo tenemos que respetar porque somos de ahí, así es así son los usos y costumbres de nuestro municipio. ${ }^{8}$

En sus definiciones del buen vivir los jóvenes retoman las ideas y los valores expuestos por los mayores, reafirmando una definición de kuxlejal como vivir de acuerdo con los usos y costumbres. En este sentido el ejercicio intergeneracional e interétnico puso en evidencia cómo en este municipio los cargos cumplen una función clave en la formación de las nuevas generaciones hacia la continuidad de los valores y la identidad. En la permanencia del sistema de cargos está entonces la posibilidad de perpetuar y transmitir un estilo de vida que caracteriza la autoimagen tradicional como andreseros.

\section{LEKIL KUXLEJAL Y SISTEMA DE CARGOS: HACIA UN ANÁLISIS DE LOS DATOS}

Ahora bien, una vez presentados los resultados del foro, quisiéramos proponer una interpretación que nos permita explicar por qué en el municipio de estudio, a diferencia de las concepciones aymara y kichwa de buen vivir, así como de la misma perspectiva tseltal del lekil kuxlejal, más enfocadas en el tema de las relaciones entre hombre y naturaleza, las autoridades hayan enfatizado más el aspecto del respeto entre seres humanos, mismo que se aprende de la experiencia de vivir y respetar los cargos.

En otro artículo (Ortelli 2012) se avanzó la hipótesis de que la peculiaridad de la historia del municipio, y particularmente la habilidad política de sus autoridades de gobernar para un buen vivir, justificaban y explicaban la concepción local sobre el kuxlejal, así como el énfasis que los habitantes pusieron en el conoci-

\footnotetext{
${ }^{8}$ Intervención de un joven del grupo 1.
} 
miento y mantenimiento del sistema de cargos tradicionales. Se decía ahí, que la experiencia de las divisiones políticas que han caracterizado la historia del municipio es posiblemente uno de los factores que lleva a destacar y enfatizar el aspecto ético-político del kuxlejal por encima de los demás. Finalmente se concluía argumentando que el arraigo a la cultura y tradiciones propias, lejos de ser una forma de esencialismo, parece constituir una estrategia de resistencia de los pueblos indígenas hacia las fuertes tensiones y amenazas del contexto global en el que están inevitablemente inmersos.

Una mirada más atenta nos ha llevado a profundizar este argumento, al identificar otros factores que intervienen en la formulación de una concepción ético-política del buen vivir. En este párrafo presentaremos este nuevo análisis, empezando por revisar brevemente la literatura producida sobre el sistema de cargos; después revisaremos los elementos fundamentales que caracterizan el sistema de cargos en el caso de estudio y finalmente avanzaremos nuevas hipótesis orientadas a dar una explicación más exhaustiva del tema. Es importante subrayar que la investigación está aún en proceso; por lo tanto, en este texto nos proponemos presentar las reflexiones que surgen a partir de los primeros hallazgos de investigación.

\section{ALGUNOS ANTECEDENTES TEÓRICOS SOBRE EL SISTEMA DE CARGOS}

Las características específicas de la estructura política del municipio en cuestión es organizada alrededor de una jerarquía político-religiosa, conocida como sistema de cargos.

En relación con la definición, Carrasco la denomina un tipo de organización política y ceremonial cuyas características ${ }^{9}$ presentan numerosas variantes locales. En este sentido los rasgos fundamentales de la estructura, según Carrasco (1990), son: el escalafón y el patrocinio individual. Korsbaek nos recuerda que existe la opinión general entre los antropólogos de que se trata de un «inven-

\footnotetext{
9 Los rasgos que el autor identifica son: el hecho que los cargos constituyen una jerarquía ordenada conforme rango y línea de autoridad; es un sistema tradicional o consuetudinario separado generalmente del constitucional o legal, los puestos son por un año, no hay reelección; los cargos están en escala y deben ocupar un orden determinado y después del superior se alcanza el grado de anciano o principal; la escala combina puestos civiles y religiosos que los participantes alternan; la participación en la escala de cargos está abierta a todos los miembros varones de la comunidad; en comunidades divididas en barrios hay alternancia de puestos entre los representantes, hay patrocinio individual de las funciones públicas; los gastos sustraen recursos pero aumentan el prestigio del patrocinador.
} 
to antropológico» (2009:39), que data de 1937, año en que se publica una de las primeras descripciones ${ }^{10}$ de la institución por Sol Tax, y que posteriormente Korsbaek (2009) ha profundizado y ampliado, categorizándola, para el caso de las comunidades mesoamericanas, como «típico sistema de cargos»:

el sistema de cargos consiste en un número de oficios que están claramente definidos como tales y que se rotan entre los miembros de la comunidad quienes asumen un oficio por un periodo corto de tiempo después de lo cual se retiran a su vida normal por un largo período de tiempo. Los oficios están ordenados jerárquicamente y el sistema de cargos comprende a todos — o a casi todos- los miembros de la comunidad. Los cargueros no reciben pago alguno durante su período de servicio, por el contrario muy a menudo el cargo significa un costo considerable en tiempo de trabajo perdido y en gastos en dinero en efectivo, pero como compensación el cargo confiere al responsable un gran prestigio en la comunidad. El sistema de cargos comprende dos jerarquías separadas, una política y una religiosa, pero las dos jerarquías están íntimamente relacionadas y después de haber asumido los cargos más importantes del sistema un miembro de la comunidad es considerado como pasado o principal (Korsbaek 2009:41).

Ahora bien, si analizamos el sistema de cargos hoy en el municipio de San Andrés podemos identificar muchos de los rasgos señalados por Korsbaek, pero a la vez emerge un aspecto tal vez poco analizado, a saber, la flexibilidad de la institución y su capacidad de transformación, características que resultan de su historicidad.

Desde un principio los investigadores reconocieron la enorme variabilidad de formas que asume el sistema de cargos, a partir de las cuales se ha producido inmensa cantidad de textos y perspectivas sobre el tema. Para sintetizarlas Castro (1994) propone agruparlos a partir de cinco enfoques teóricos explicativos.

El primero ${ }^{11}$ es un acercamiento descriptivo y sienta las bases para estudios sucesivos que permiten entender los mecanismos de legitimación y manipulación del poder en las sociedades indígenas, diferenciándolos de los que operan en la sociedad nacional. La perspectiva de la comunidad como espacio armónico y

${ }^{10}$ De hecho Korsbaek comenta que en realidad la primera descripción se encuentra en un artículo de Noriega Hope de 1922, pero debido a su poca difusión el texto quedó en el olvido.

${ }^{11}$ Los autores que lo integran son: Tax (1937); Villa Rojas (1947), Cámara (1952, Guiteras (1961), Pozas (1977) y Aguirre Beltrán (1953). Citados en Castro, 1994:505. 
homogéneo como telón de fondo de los trabajos lleva a considerar el sistema de cargos en los términos de mecanismo regulador del equilibrio intracomunitario.

El segundo enfoque ${ }^{12}$ asume el sistema de cargos como un mecanismo de defensa y protección de la comunidad ante la explotación del exterior. Se resalta la función compensatoria del sistema como nivelador de riqueza, que en su función política impediría la monopolización del poder. Esta perspectiva se basa en la idea de la «comunidad corporativa cerrada» (Wolf 1957) como entidad que resiste y que protege de las amenazas externas, sin tomar en cuenta las múltiples interrelaciones de las comunidades con el contexto más amplio en el que se insertan.

En contraposición a este enfoque surge el tercero, que tiene como principales exponentes Harris (1964) y Cancian (1989). De acuerdo con Castro (1994) el primero lleva el análisis a un contexto más amplio que rebasa el espacio comunitario insertándolo en una dinámica regional más amplia. En este sentido Harris (1964) cuestiona la función defensiva y homogeneizadora del sistema de cargos, que define una institución «represiva y abusiva» establecida en las comunidades indígenas por los curas católicos (Chanse y Tylor 1985). En la misma tónica, aunque sin romper completamente con la perspectiva funcionalista de Wolf y Nash, Cancian (1989) ${ }^{13}$ profundiza la función estratificadora del sistema de cargos y llega al extremo de sostener que dicha institución opera en la comunidad como un sistema de clases sociales semejante al estadounidense.

Las dos perspectivas anteriores han sido ampliamente debatidas y de alguna manera constituyen un punto de partida para el desarrollo de una cuarta perspectiva de estudios sobre el sistema de cargos que — subraya Castro (1994)—, nos lleva a interpretarlo como un proceso más que como una categoría. Los autores ${ }^{14}$ que la integran enfatizan la importancia de los contextos a partir de los cuales se debe leer el sistema de cargos, cuyas funciones cambian dependiendo de la coyuntura específica en la que se estudie: desde esta perspectiva puede ser un mecanismo igualador o estratificador, de defensa o explotación. En particular Chanse

12 Los autores que lo integran son: Wolf (1957); Nash (1958); Carrasco (1961); Hermitte (1970); Verbitski (1961), Zábala (1961), Nash (1970) y Dow (1974). Véase Castro (1994:506).

${ }^{13}$ Es importante recordar que la investigación de Cancian se realiza en el marco del Proyecto Harvard «El hombre y la naturaleza», que, junto al Chicago-Harvard Proyect y el Instituto Lingüístico de Verano se hacen portadores del proyecto de aculturación promovido por el Estado mexicano. En este contexto también cabe mencionar que la metodología usada por los investigadores en el levantamiento de los datos de campo debe ponernos en alerta sobre los resultados encontrados. Seminario del doctor Fábregas, México, 11-16 de febrero de 2013.

${ }^{14}$ De acuerdo con el autor se integra por los trabajos de Castaingts (1979), Chance (1985), Greenberg (1981), Rus (1980), Tylor (1985) y Wasserstrom (1983). Castro 1994. 
y Tylor (1985) cuestionan las miradas teóricas «atemporales» (1985:20) que ven el origen del multicitado sistema en el siglo XVI y la persistencia de su estructura y funciones hasta el presente. Por medio de un amplio análisis histórico comparan cuatro regiones mexicanas ${ }^{15}$ separando tres componentes: la jerarquía civil, la jerarquía religiosa y la institución de la fiesta patrocinada por individuos; y muestran así que la jerarquía cívico-religiosa moderna es más el producto de los cambios producidos en el siglo XIX que una adaptación colonial (Chanse y Tylor 1985).

Desde la misma perspectiva Rus y Wasserstrom (1980) defienden la tesis del surgimiento del sistema de cargos a finales del siglo XIX y principios del XX, como respuesta al desarrollo económico y a los cambios demográficos. Asimismo, consideran que dicha institución ha cambiado forma y funciones dependiendo del papel que hombres y mujeres locales han jugado en estas transformaciones, como muestra la comparación entre Zinacantán y Chamula en cuyos casos los actores locales tienen diferentes respuestas frente al crecimiento de la agricultura comercial en el centro de Chiapas. A partir de esto los autores demuestran que, lejos de ser una pared de defensa del tradicionalismo, la jerarquía cívico-religiosa parece haber surgido en aquellas comunidades en las que los habitantes fueron proyectados en las relaciones de clase que se desarrollaron en toda la región (Rus y Wasserstrom 1980:467). Esta perspectiva enfatiza un aspecto tal vez poco analizado, a saber, la flexibilidad de la institución y su capacidad de transformación, características que resultan de su historicidad y que en el caso de estudio son particularmente interesantes. Como señala Korsbaek (2009), Rus y Wasserstrom son los primeros autores que colocan el sistema de cargos en Chiapas en una dimensión temporal, y que otorgan a los pueblos indígenas y sus instituciones vitalidad en lugar de pasividad.

Una quinta perspectiva ${ }^{16}$ se enfoca a explicar la visión mesoamericana e incaica del mundo, y que parte de la idea de que la fiesta del sistema de cargos es un campo de interrelación social y un mecanismo que refuerza la identidad comunal y la cohesión intragrupal. Los autores señalados también estudian la fiesta como «campo de lucha simbólica» en la que se manifiestan las contradicciones existentes entre los grupos de poder que controlan los recursos de la comunidad. En este sentido, los rituales del sistema de cargos son «estrategia ritual de poder»y sirven para legitimar, desarrollar y mantener el poder (Castro 1994:511).

15 Jalisco, centro de México, Valle y Sierra zapoteca de Oaxaca (Chanse y Tylor 1985).

${ }^{16}$ Los autores son Montes Castillo (1989); López Austin (1990) y Galinier (1990). Citados en Castro 1994. 
Para completar este recorrido sobre las perspectivas teóricas que han intentado explicar la institución del sistema de cargos, me parece importante mencionar la perspectiva que ve en el sistema de cargos una versión moderna de las sociedades de realeza sagrada, definida por Dehouve (2006:23) «[...] como una forma de sociedad caracterizada por su relación particular entre el campo político y el religioso a través de la figura de un personaje central o un grupo de 'personajes reales' con la facultad de garantizar la prosperidad del grupo social por su actividad ceremonial». De acuerdo con esta autora, lo que define la realeza sagrada es el tipo de funciones ejercidas por la persona en el poder y el vínculo que ella establece entre los campos político y religioso.

Dada la importancia de la vinculación entre el ámbito político y el religioso en el caso de estudio que me propongo analizar, considero necesario destacar algunos aspectos de esta perspectiva. Comparando el estudio de cargos en la comunidad tlapaneca de Guerrero con una relectura de las monografías clásicas de Guiteras (1961) y Pozas (1977) sobre tres municipios chiapanecos ${ }^{17}$ y la descripción de Piedrasanta (2003) sobre los chuj de la Sierra de los Cuchumatanes en Guatemala, la autora profundiza el carácter sagrado de los cargos, independientemente de su naturaleza (religiosa o política). En este sentido, volviendo la vista a teorías clásicas (Frazer 1961 y Hocart 1936), asume la realeza sagrada como un tipo distinto de la de derecho divino y la monarquía absoluta, ya que califica una forma especial de organización social en la que el centro es un personaje reputado responsable de la prosperidad de su pueblo así como de su desdicha. A partir de esta idea, el análisis comparativo entre los diferentes sistemas no puede limitarse a la comparación de los nombres de los cargos, sino tomar en cuenta la distinción entre función-tarea y función-cargo, siendo las primeras las que definen la existencia de una sociedad de realeza sagrada, a saber, las funciones penitenciales, ceremoniales, sacerdotales y coercitivas. Aplicando el modelo clásico de realeza sagrada a las comunidades indígenas mesoamericanas, Dehouve (2006) propone considerar al presidente municipal como la figura central del sistema de cargos, rescatándolo del papel de cargo impuesto que se le había asignado. Al grupo central que gira alrededor de este se agrega una constelación de grupos periféricos que lo replican, manteniendo y reproduciendo el carácter sagrado de los cargos. La dinámica de la realeza sagrada hace que los puestos se desplieguen sin perder su carácter. Esta perspectiva permite, de

${ }^{17}$ En el caso de Guiteras se trata de Cancuc y Chenalhó; en el caso de Pozas de San Juan Chamula. 
acuerdo con la autora, comparar las diferentes formas de sistemas como variantes de uno mismo, el de realeza sagrada, definido a partir de la unidad entre los campos político y religioso.

\section{EL SISTEMA DE CARGOS EN SAN ANDRÉS LARRÁINZAR HOY: HACIA UNA INTERPRETACIÓN}

El sistema de cargos en el municipio de estudio ha sido explicado y analizado por diferentes autores. Si comparamos las descripciones de la organización política que estos han dejado sobre el municipio de Larráinzar, podemos observar la flexibilidad de esta institución, que se viene adaptando a las diferentes coyunturas y procesos socio-históricos. Este es un aspecto muy relevante que me interesa resaltar, ya que manifiesta el dinamismo de una institución, cuyas características con el tiempo se han venido modificando con respecto a las primeras descripciones que dieron origen a la definición del típico sistema de cargos chiapaneco. Los autores que la han analizado (Holland 1963, Ochiai 1985, Gorza 2006) se han enfocado en una línea descriptiva de la estructura, y una lectura comparativa de sus textos nos permite analizar cómo ha ido cambiando a lo largo de 50 años.

Holland (1963) describe la jerarquía político-religiosa de San Andrés como compuesta por tres cuerpos: el Ayuntamiento Constitucional, el Ayuntamiento Regional y los principales. «El primero es la única organización administrativa reconocida por el gobierno del estado de Chiapas, y no tiene ninguna relación con los asuntos religiosos. El Ayuntamiento Constitucional y el Ayuntamiento Regional están sólidamente integrados porque los puestos del primero equivalen a los puestos políticos más altos del segundo» (1963:57).

El principio jerárquico es el de las generaciones que, como escribe el autor,

[...] es tan importante en las relaciones de parentesco entre los tzotziles, que se infiltra en todos los planos de su percepción de la realidad: desde el punto de vista indígena, son bankilal o its'inal18 los santos, las cuevas, los charcos; las montañas mismas están sujetas a este orden de importancia (Holland 1963:49).

Otras características importantes para integrarse a la jerarquía político religiosa son: la experiencia, el conocimiento ritual y esotérico, los poderes sobrenaturales (nagual poderoso), cualidades personales como inteligencia y aptitudes admi-

\footnotetext{
${ }^{18}$ Del tsotsil 'hermano mayor' y 'hermano menor'. Holland 1963.
} 
nistrativas y, por supuesto, la solvencia económica. Es interesante notar cómo la forma de control que los ladinos establecen sobre estas jerarquías se da a través de los cargos de secretario ladino y sacerdote ladino, que «forman parte de los lazos que unen al municipio con el estado y con las jerarquías nacionales y sus instituciones respectivas» (Holland 1963:63).

En 1982, Ochiai (1985) observa que la organización política de Larráinzar está compuesta por dos cuerpos políticos y administrativos: el Ayuntamiento Constitucional y el Regional, ambos operantes en la cabecera municipal. A diferencia de la estructura analizada por Holland (1963), emerge una clara separación entre las funciones de ambos, así como el cambio de papel de los ladinos, que se convierten en meros funcionarios al servicio del Ayuntamiento integrado por autoridades tsotsiles, como resultado del proceso de expulsión de los jkaxlanetik en 1974. Según Ochiai, el Ayuntamiento Constitucional está compuesto por 13 jóvenes bilingües ${ }^{19}$ dirigidos por el presidente municipal y los cargos se eligen en parte por elección y en parte por nombramiento. Sus funciones fundamentales conciernen a la administración del presupuesto, la planificación de obras públicas, la solicitud de recursos al gobierno además del arbitraje en las disputas, la organización de las elecciones, la recolección de impuestos y contribuciones para las fiestas, las relaciones con el gobierno del estado y la supervisión de los trabajos públicos. El Ayuntamiento Regional está compuesto por 29 miembros categorizados jerárquicamente y llamados servidores por un año (j-abteletik ta jun ja'bil) y cuyas funciones dependen del cargo específico que cumplen. De ese modo, el autor señala que los escribanos son solteros bilingües, recolectan impuestos en el mercado y acompañan al presidente municipal; ${ }^{20}$ los mayoles trabajan como policías y mensajeros y los demás funcionarios, portadores del bastón y funcionarios del cabildo, además de ayudar a los funcionarios del Ayuntamiento Constitucional en labores administrativas, tienen fundamentalmente atribuciones rituales. Finalmente el autor describe la jerarquía religiosa como compuesta por tres categorías: los encargados de la iglesia (dos fiscales, dos sacristanes ${ }^{21}$ y 27

${ }^{19}$ Consideramos que esta información tiene que ser profundizada porque contradice lo que observamos en relación con el espacio que ocupan los jóvenes en el sistema de cargos actual.

${ }^{20}$ En realidad nos sorprende la tajante clasificación del autor sobre las características de los escribanos como solteros bilingües, así como su función como acompañantes del presidente municipal, ya que como integrantes del Ayuntamiento tradicional, más bien acompañan a los alcaldes, gobernadores, mayoles y regidores tradicionales.

${ }^{21}$ En este caso también la categoría de sacristanes nos resulta curiosa, porque se trata únicamente de fiscales. 
mayordomos), los anfitriones de las fiestas (alféreces y ayudantes) y los especialistas rituales (músicos, capitanes y danzantes) (Ochiai 1985). Más allá de la descripción de la estructura, como señala Korsbaek (1992), el estudio de Ochiai, enfocado al «ritual público», contribuye al análisis de los sistemas de pensamiento, y ayuda a comprender los mecanismos culturales de motivación y legitimación.

La estructura de cargos descrita es retomada unos años después por Gorza (2006), quien en su etnografía ofrece una descripción minuciosa de cómo los habitantes de Larráinzar "habitan²2 el tiempo y el espacio» (2006:273). Este autor describe los procesos de reconfiguración de la identidad y el territorio, narrando el municipio a través de la descripción de sus paisajes naturales y humanos. En este recorrido, uno de los espacios habitados es el poder y el autor se enfoca particularmente en el sistema de cargos, sus crisis y las tensiones que surgen por la coexistencia de principios jerárquicos e igualitarios que operan a nivel del Ayuntamiento tradicional y el Gobierno constitucional, a su vez acompañados por una jerarquía religiosa especializada en el culto de los santos. La estructura del Ayuntamiento Constitucional se ha ido complejizando y especializando respecto de la que describe Ochiai, mientras que la estructura del Ayuntamiento Regional y la jerarquía religiosa no han cambiado mucho desde 1985. Por ejemplo Gorza no refiere la presencia de sacristanes en la jerarquía de la Iglesia, sino únicamente de fiscales, situación que también observamos actualmente:

Llevar un cargo mantiene todavía hoy el antiguo sentido, un privilegio y una carga que se traducen en la tarea de garantizar el camino de la comunidad con gastos propios. El orden tiene siempre que ser restablecido, porque la supervivencia del mundo está constantemente amenazada. Para evitar estos riesgos es necesario confiar en la sabiduría de los ancianos y en la participación coral de la población en la «cosa pública» (Gorza 2006:187).

A diferencia de los autores mencionados antes, Gorza ahonda en el tema a partir del análisis de un documento fechado 12 de abril de 1981, en el que 2000 personas procedentes de diferentes parajes del municipio manifiestan las razones de su descontento hacia el sistema de cargos, pidiendo ser «liberados» de la obligación de cumplir con los cargos por el costo elevado que estos implican

${ }^{22}$ Como menciona el autor «el concepto de habitar no indica solo la condición de arraigamiento físico en un lugar, sino los procesos que transforman los territorios en paisajes culturales y los mapas mentales que configuran y que son producidos por tierras, historias y redes comunicativas particulares» (Gorza 2006:273). 
para las familias. Entre las razones que justifican esta demanda está el peso excesivo de las cooperaciones, el hecho de que no se tomen en cuenta las características socioeconómicas de las familias, además de la obligatoriedad del cargo. El documento se refiere a los cargos religiosos, a saber, alférez, capitán, mayordomo, alcalde juez, stacobil jkuchel y pasión. Es interesante observar cómo en el caso estudiado, a pesar de estos antecedentes y frente a la amenaza constante de su desaparición, el sistema se mantiene y, lejos de ser una estructura rígida, logra adaptarse a las situaciones cambiantes que vive el municipio.

\section{LOS CARGOS EN LA SAN ANDRÉS DE HOY}

En la actualidad la estructura de los cargos no es muy diferente de la que describió Gorza en 2006. El Ayuntamiento Constitucional se compone de 15 integrantes ${ }^{23}$ que duran en el cargo tres años. Las decisiones se toman de manera colectiva en el cabildo, donde el presidente, el síndico, el primer regidor y el tesorero se consultan con los regidores antes de tomar las decisiones. En los últimos años la presencia de empleados contratados por el Ayuntamiento Constitucional ha ido complejizando la estructura, ampliando la participación de jóvenes profesionistas, hombres y mujeres que apoyan al presidente en la dirección de proyectos, la operación del programa Oportunidades, la contabilidad y la gestión de obras de infraestructura. En particular, a partir de 2007 se da un cambio importante a raíz del nombramiento como presidente de un profesionista, ${ }^{24}$ licenciado en derecho, quién empieza a promover la inclusión de jóvenes profesionistas andreseros en el Ayuntamiento Constitucional, cambio que a últimas fechas tuvo un impulso mayor con el actual titular, egresado de la Universidad de Chapingo.

El Ayuntamiento Regional cuenta con 29 miembros categóricos ${ }^{25}$ cuyo cargo dura un año y no ha registrado modificaciones desde la descripción de Gorza en 2006, excepto que los escribanos no son solteros bilingües como señalaba Ochiai (1982). Los cargos civiles se alternan los respectivos religiosos que se dividen en tres categorías: encargados de la iglesia, cuatro fiscales y 27 mayordomos

\footnotetext{
${ }^{23}$ Los cargos son presidente, síndico y suplente, tesorero, juez y suplente, seis regidores y tres suplentes.

${ }^{24}$ Hacemos una distinción entre los maestro, que ya en años anteriores han ocupado el cargo de presidente y los profesionistas que han egresado de una carrera universitaria. Aquí nos referimos a estos últimos.

${ }^{25}$ Son dos gobernadores y tres alcaldes, un síndico tradicional, 11 regidores, 10 mayoles y dos escribanos.
} 
divididos entre 13 mayores y 14 menores; especialistas rituales: nakanvanej, seis capitanes, músicos tradicionales y larineros, cuatro auxiliares de capitanes (sta jobil cuchel); ${ }^{26}$ anfitriones de la iglesia (alférez), dos paxiones para el Carnaval. En Semana Santa se agregan 12 apóstoles y 12 soldados.

Es importante señalar que los miembros del Ayuntamiento Regional, desde hace aproximadamente 25-30 años reciben honorarios, y desde 2007 también los integrantes de la jerarquía religiosa son apoyados con una cantidad mínima que les sirve para cubrir costos y viáticos. Además, reciben apoyo de refresco, incienso y transporte para las fiestas. De hecho, hay una participación importante del Ayuntamiento Constitucional en las actividades religiosas organizadas por la jerarquía religiosa y el Ayuntamiento tradicional.

A esta estructura cabe agregar a otros sectores: el Comité de partido y el Comisariado de bienes comunales, ambos integrados por 12 miembros, la Confederación Nacional Campesina, compuesta por seis integrantes y los Frentes juveniles, con 11 en la cabecera y un representante en cada localidad. Se trata de otro tipo de servicios que se prestan a la comunidad y se ocupan de problemas específicos como las tierras, conflictos políticos o actividades juveniles. La particularidad de estos cargos radica en que, excepto en los frentes juveniles, son asignados a personas que ya tienen una trayectoria en los cargos y son pasados. Participan en la elección de los cargos más relevantes y en el nombramiento de los empleados que en cada periodo son contratados por el Ayuntamiento Constitucional. A pesar de no crearse una línea fija de cargos, se trata de un servicio a través del cual las personas acumulan méritos que les permiten sucesivamente alcanzar otros cargos relevantes.

Si nos quedamos en el análisis de la estructura, podríamos decir que tendemos a una perspectiva estática de los cargos y perdemos de vista las pequeñas transformaciones y adaptaciones que se dan constantemente en su funcionamiento, aun cuando se busca mantener inalterada la lógica de la institución. En este sentido es importante identificar los principios que la caracterizan. Aunque exista un deber ser del camino que deben recorrer en los cargos, en la práctica existen más posibilidades para recorrerlo y en esta variedad radica la flexibilidad y adaptabilidad del sistema de los andreseros a los continuos retos que impone la modernidad, como la incorporación de las mujeres, los profesionistas, los migrantes retornados, otras religiones y partidos políticos.

\footnotetext{
${ }^{26}$ Solo para tres fiestas: San Andrés, Virgen de Concepción y Virgen de Guadalupe.
} 


\section{LA LÓGICA DE LOS CARGOS}

Analizando más profundamente el significado de las palabras de las autoridades, podemos decir que, más allá de las estructuras, el sistema de cargos garantiza un estilo de vida que caracteriza la identidad como andreseros. En este estilo de vida cabe destacar las dimensiones y valores que vienen a enfatizar los cargos.

Uno de los elementos importantes a resaltar es la preeminencia del cargo sobre el individuo que lo ocupa, como menciona Korsbaek (1992:14), «Es el cargo que gobierna, no la persona que lo ocupa». En efecto, en el caso de estudio la toma del cargo se da a través de un largo proceso ritual solemne y colectivo que involucra, por lo menos en el caso de los mayordomos, el rezo, la transmisión simbólica del poder por medio de una regla de madera que representa la cruz y su asunción con el simbólico cambio de ropa por prendas nuevas. Y a partir de ese momento la persona ya no se identifica con su nombre propio, sino con el del cargo que ocupa. ${ }^{27} \mathrm{La}$ relatividad del individuo ${ }^{28}$ se manifiesta también en razón de que el cargo involucra a la esposa, los hijos y la familia extensa, quienes además de colaborar en las fiestas que le tocan al carguero deberán observar un comportamiento recto y socialmente adecuado. Por otra parte, aunque el individuo se diluye en el cargo, cabe mencionar que las cualidades personales para su ejercicio son importantes porque influyen en la evaluación que se hace de su desempeño, así como en la posibilidad de seguir en el camino de los cargos. Es así que, a pesar de la temporalidad, que representa un servicio que se presta a la comunidad, cada uno significa un escalón más en un sistema jerárquico basado en el principio gerontocrático de mayor a menor y preeminencia de lo masculino sobre lo femenino. En este sistema los cargos son una forma de acceso a lo que podríamos definir como una ciudadanía dosificada, ${ }^{29}$ pues cada uno implica un proceso

${ }^{27}$ Cabe mencionar que en cada tipología de cargo hay ciertas diferencias en la forma de llevar a cabo el ritual; aquí me refiero a la transmisión del cargo del mayordomo mayor, en el que el mayordomo nuevo recibe las pertenencias del Santo con la dotación de velas en un largo ritual que dura una noche y un día, tras rezos, ahumaciones y transmisión pública del poder.

${ }^{28}$ Retomando a Dumont (1970), el concepto de individuo tiene una doble vertiente: una, que corresponde a la construcción occidental, está más enfocada al yo individual como espacio interno de sentimientos, emociones, libertad y que se caracteriza la posibilidad de elección y decisión como uno de los derechos fundamentales; la otra que corresponde al yo como persona y es la vertiente del individuo inmerso en la sociedad (Dumont 1970).

${ }^{29}$ Cuando hablamos de ciudadanía retomamos la definición de Costa, como la «relación entre un individuo y cualquier orden que lo gobierna y se da en términos de expectativas, pretensiones, derechos, deberes, modalidades de pertenencia y criterios de diferenciación, estrategias de inclu- 
de aprendizaje y conquista de derechos y obligaciones que tiende a un orden creciente y que culmina con el cumplimiento del último cargo tradicional. Es el servicio a la comunidad que otorga derechos ciudadanos al carguero y su familia y se caracteriza por la existencia de múltiples niveles que implican derechos y obligaciones diferentes, dependiendo de la edad, el sexo, el credo religioso y la filiación política. El cargo no se busca ni se desea, sino se recibe por designación de la comunidad. Como menciona el presidente municipal, la persona que recibe un cargo debe tener estas cualidades:

Primero la trascendencia, cómo ha estado atendiendo a la gente, cómo ha desempeñado los cargos anteriores, si ha sido responsable... pues eso cuenta mucho, cuenta muchísimo. Pues el trabajo que uno hace; los cargos es por mérito. Así es y pues la gente es muy observadora, observa a la gente cómo platica, cómo atiende... Eso es lo que toman en cuenta. ${ }^{30}$

A esto es importante agregar que la persona debe vivir en el municipio y ser evidentemente originaria de ahí.

Existe un camino tradicional, que empieza con el cargo de mayordomo, pasa por regidor tradicional o síndico y termina como primer alcalde, ${ }^{31}$ último cargo que lleva al estatus de principal o nakanhuanej. ${ }^{32}$ Los principales juegan un papel muy importante porque se reúnen, rezan, dialogan, vigilan y buscan las maneras de resolver los problemas.

El camino ideal de los cargos puede que se desvíe y modifique su curso, como ejemplifica el caso del actual primer regidor constitucional, quien empezó su camino en el sistema a los 27 años como mayordomo menor y hasta hace seis años siguió la línea tradicional hasta llegar a segundo alcalde. Ya a punto de jubilarse, como él mismo menciona, se desvió al camino constitucional y actualmente fue

sión y exclusión» (Costa 2005:4). Desde esta perspectiva los andreseros son ciudadanos a partir de dos dimensiones, a saber: a) la relación con el orden político-jurídico local encarnado en el sistema de cargos y b) la relación con el Estado. Es en relación con el orden político local que propongo el concepto de ciudadanía dosificada, ya que, a diferencia de la ciudadanía al estilo occidental, se trata de un proceso progresivo de obtención de derechos ciudadanos, que dura por la gran parte de la vida de las personas e implica su activa participación al servicio de la comunidad.

${ }^{30}$ Entrevista con el presidente municipal constitucional de San Andrés Larráinzar, 14 de febrero de 2014.

${ }^{31}$ Entrevista con el primer regidor constitucional de San Andrés Larráinzar, 17 de abril de 2014.

32 El nakanhuanej es el consejero ritual, que llega en las fiestas, sabe rezar, prepara y sirve la comida a los invitados y conoce todos los aspectos del ritual. 
nombrado primer regidor constitucional. Como podemos observar en la gráfica 1, siguió su camino alternando el cargo a breves momentos de descanso y cumpliendo con los de alférez complementarios a los cargos tradicionales. En su vivencia personal hubo un descanso de diez años por cuestiones de salud, luego volvió al camino, hasta que se desvió al Ayuntamiento Constitucional con el nombramiento de juez. Como él mismo relata, cada cargo es un aprendizaje diferente en el que la experiencia y el desempeño constituyen la posibilidad de seguir sirviendo con un cargo mayor, hasta completar el ciclo y obtener así una ciudadanía plena.

\section{LOS CARGOS COMO ESCUELA}

Cada cargo representa una oportunidad de aprendizaje en una suerte de pedagogía metonímica y vivencial en la que la observación, la imitación y la repetición son los elementos fundamentales en los que se rige el proceso. El principio gerontocrático de mayor a menor es la columna vertebral de un sistema en el que el respeto hacia los mayores es un valor fundamental. En cada reunión y ritual las autoridades dedican mucho tiempo al saludo, que representa simbólicamente la jerarquía mayor-menor. Es importante resaltar que, independientemente de la importancia del cargo que la persona ocupa, ésta debe respeto a un mayor inclinando su cabeza y dejándola tocar por el mayor. Asimismo personas de un

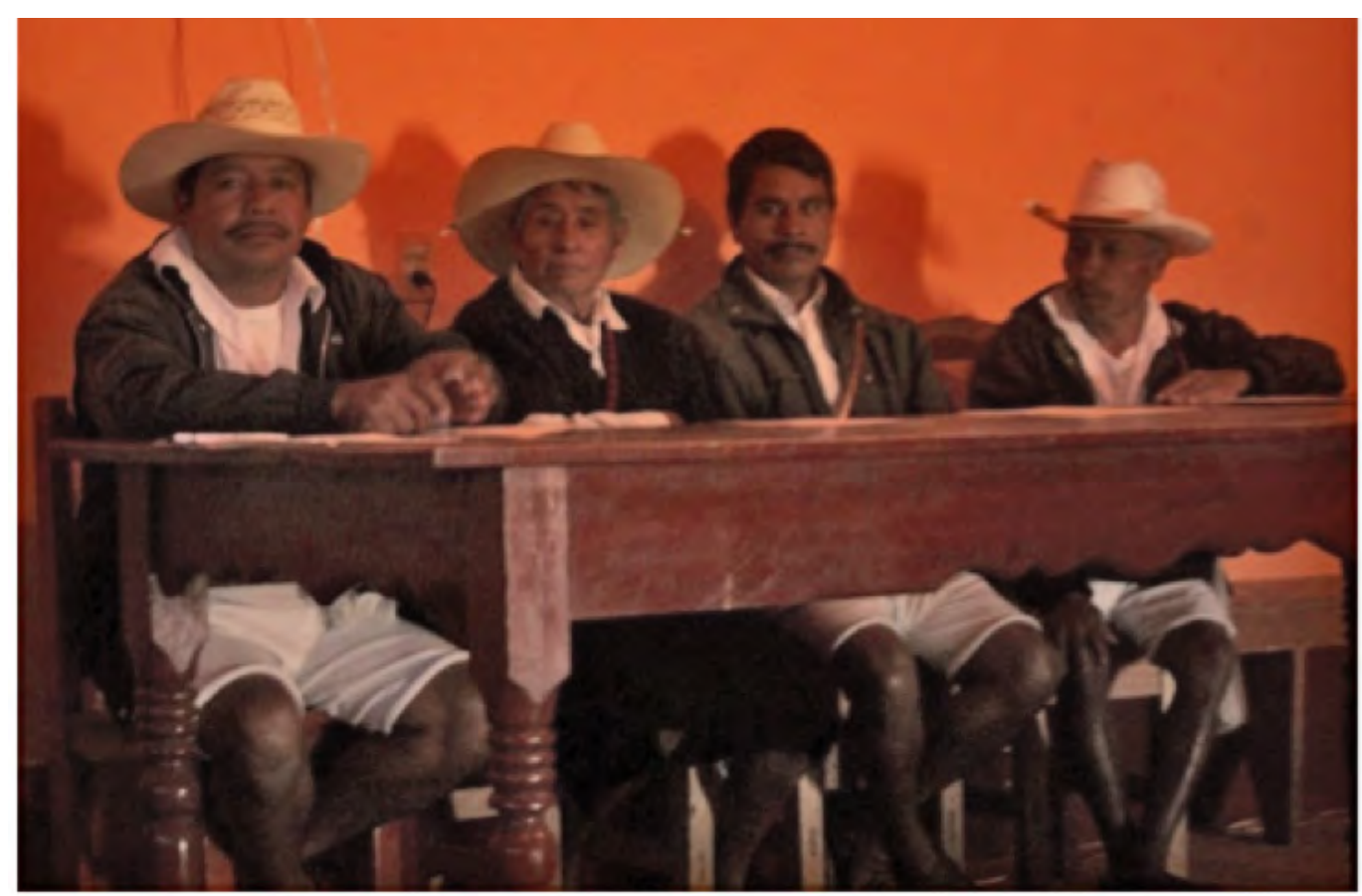


mismo rango de edad se saludan dándose la mano en signo de igualdad. Ahora bien, en la forma tradicional de los cargos, el tipo de cargo ocupado también correspondía a cierto rango de edad, con el resultado que la mayor edad siempre correspondía a un cargo de mayor importancia. Esto está cambiando actualmente a raíz de la incorporación de los profesionistas en el sistema, mismo que empiezan a ocupar cargos de gran importancia siendo muy jóvenes, pero que siguen respetando el principio gerontocrático de mayor a menor.

La importancia del reconocimiento del mayor como persona de mayor experiencia de la que se aprende es particularmente evidente en el caso de la jerarquía religiosa, que es el lugar donde los andreseros dicen que «empieza todo». La entrada tradicional al sistema de cargos empieza de hecho con el nombramiento como mayordomo menor (vikit martoma), que hacen los fiscales (pixcaletik) en calidad de mayor autoridad de la Iglesia. Es el mayordomo saliente quién tiene la encomienda de buscar a su reposición y proponerla a los fiscales. Una vez que el nuevo mayordomo acepta, después de la o las visitas que le hacen los fiscales, empieza un proceso de enseñanza-aprendizaje por el mayordomo saliente, quien cada ocho días por seis meses se reúne con él para enseñar el rezo y las actividades. El nuevo mayordomo aprende observando e imitando al anterior, hasta que está listo para ejercer el cargo. Una vez en el cargo el mayordomo tiene un año de aprendizaje en el que convive con los demás mayordomos y aprende del mayordomo mayor (its'inal mol) que los encabeza.

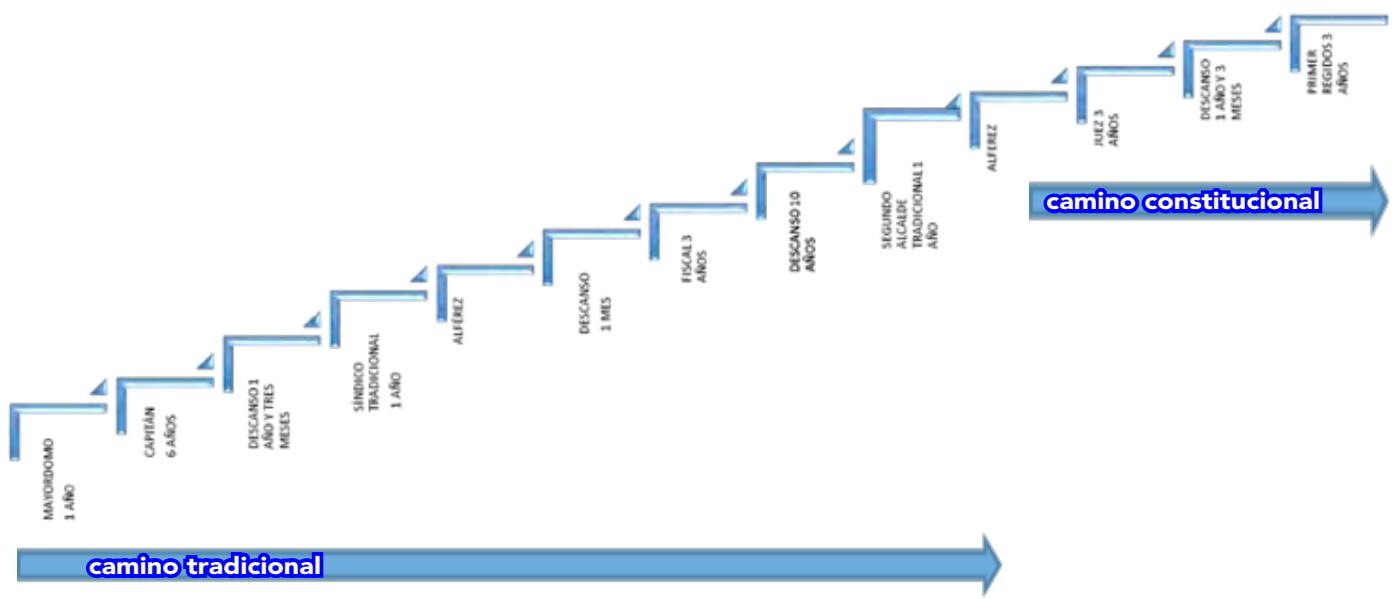

Gráfica 1: Camino de cargos del actual Primer Regidor Constitucional

Fuente: elaboración propia a partir de los datos de campo. 
En el caso de los dos mayordomos mayores, bankilal mol e its'inal mol, el proceso de aprendizaje dura tres años. En él, primero el nuevo mayordomo mayor participa en las cuatro lavadas de ropa junto al mayor, observando los rezos y las actividades; el segundo año le toca ejercer el cargo pero en las lavadas de ropa es aún acompañado por el mayordomo mayor que ya dejó el cargo que le enseña cómo lavar y guardar la ropa del santo y el tercer año ya puede enseñar al nuevo mayordomo que lo sustituye. En el año de aprendizaje el nuevo mayordomo mayor pasa todo el tiempo parado o sentado atrás del mayordomo mayor saliente, observándolo. En los demás cargos tradicionales y hasta constitucionales, la persona que ejerce el cargo siempre es parte de un grupo y, aun cuando tiene comisiones personales que ejercer, aprende constantemente de los mayores de su grupo.

La importancia del reconocimiento del mayor como persona de más experiencia de la que se aprende es particularmente evidente en el caso de la jerarquía religiosa, que es considerado por los andreseros como el lugar donde «empieza todo». La entrada tradicional al sistema de cargos inicia de hecho con el nombramiento como mayordomo menor (vikit martoma), que hacen los fiscales (pixcaletik) en calidad de mayor autoridad de la Iglesia. Es el mayordomo saliente quien tiene la encomienda de buscar a su relevo y proponerlo a los fiscales. Una vez que el nuevo mayordomo acepta, después de una o más visitas que le hacen los fiscales, empieza un proceso de enseñanza-aprendizaje por el mayordomo saliente, con quien cada ocho días, por seis meses, se reúne para que aprenda el rezo y las actividades. El nuevo mayordomo asimila observando e imitando al anterior, hasta que está listo para ejercer el cargo. Una vez en el cargo tiene un año de aprendizaje en el que convive con los demás mayordomos y aprende del mayordomo mayor (its inal mol) que los encabeza.

En el caso de los dos mayordomos mayores, bankilal mol e its'inal mol, el proceso de aprendizaje dura tres años. En el primero, el nuevo mayordomo mayor participa en las cuatro lavadas de ropa junto al mayor, observando los rezos y las actividades; el segundo año le toca ejercer el cargo pero en las lavadas de ropa es aún acompañado por aquel, que para entonces ya dejó el cargo y le enseña cómo lavar y guardar la ropa del santo; y el tercer año ya puede enseñar al nuevo mayordomo que lo sustituye. En el año de aprendizaje el nuevo mayordomo mayor pasa todo el tiempo parado o sentado atrás del saliente, observándolo. En los demás cargos tradicionales y hasta constitucionales, la persona que ejerce el 
cargo siempre es parte de un grupo y, aun cuando tiene comisiones personales que ejercer, aprende constantemente de los mayores de su grupo.

\section{CONCLUSIONES}

En este artículo nos propusimos presentar los resultados de un foro sobre el buen vivir que se llevó a cabo en el municipio de San Andrés Larráinzar, para explicar el significado y las implicaciones de una concepción del buen vivir como vivir en el respeto del sistema de cargos. Mostramos cómo, independientemente de la perspectiva ideológica o política, los cargos constituyen la ejemplificación de una forma de vivir que alimenta valores como el respeto hacia los mayores; la relatividad del individuo, que se diluye en el cargo; la temporalidad del ejercicio del poder; la rotación de los cargos; la ciudadanía como un proceso de aprendizaje progresivo en el que derechos y obligaciones son dosificados. A nivel ideal, el sistema de cargos representa para las autoridades andreseras un horizonte de valores comunes al que debieran aspirar como pueblo, sobre todo en el contexto de las crecientes y rápidas transformaciones sociopolíticas y económicas que involucran al municipio. La heterogeneidad y el pluralismo constituyen un reto cada día más grande para una estructura de poder como el sistema de cargos, cuya función se va tornando paulatinamente más ambigua. Por una parte, como institución que alimenta una serie de mecanismos que potencian los escenarios de interacción protagonizados por los miembros de la comunidad, posibilita un proyecto comunitario y, en consecuencia, un horizonte común en cuanto a valores (González 2011). Por la otra, como institución que controla y regula el acceso al poder local está bajo la presión tanto de las dinámicas locales como de las globales. En este sentido, lejos de ser una estructura estática y rígida, se compone de un conjunto de posiciones integradas y flexibles, que forzosamente deben ser capaces de adaptarse a las diferentes coyunturas histórico-políticas en las que como sistema está inmerso. Más allá de la forma que asume la estructura, lo que resulta entonces relevante estudiar son las estrategias que los actores implementan para que los valores que la rigen sigan orientando la vida de los andreseros y encuentren una vía para enfrentar el creciente individualismo fomentado por la modernidad. A partir de esta lectura resulta claro el mensaje que las autoridades andreseras quisieron transmitir en el foro, en el que pusieron énfasis en una perspectiva ético-política del buen vivir, que también está presente en el plantea- 
miento boliviano y ecuatoriano a través de la idea de armonía y equilibrio entre toda forma de existencia y del buen convivir.

Sin duda, la historia particular del municipio influye en la manera en que los andreseros conciben el buen vivir y nos lleva a reflexionar sobre el papel de contención que en este municipio ha jugado y juega la institución de los cargos frente a los conflictos religiosos y políticos que han tenido que enfrentar. Como señalan Aguilar et al. (2010), las autoridades andreseras han mostrado una gran habilidad para resolver los conflictos de manera pacífica, para mantener lo que hoy llamamos el buen vivir. En las palabras de una autoridad «aquí es diferente la gente, aquí piensa, razona, antes de actuar. $O$ sea, que si hay algo que decir, hay que decirlo con respeto, hay que plantearlo. Eso es lo que tiene Larráinzar». ${ }^{33}$ Entonces es a través de la carrera de los cargos como se aprenden los valores que guían el quehacer de las autoridades y a hablar con respeto. Es solo por medio de este sistema que puede haber un control sobre las cualidades que se consideran necesarias para ser autoridad y en este sentido nos atrevemos a plantear que, en el caso de estudio, los cargos, además de formar, constituyen a la vez una estructura de defensa, control y contención frente al individualismo y los continuos retos impuestos por la modernidad y el neoliberalismo.

\section{FUENTES DE CONSULTA}

Aguilar, Eufemio, Martín Díaz Teratol y Juan Pedro Viqueira, 2010, «Los otros acuerdos de San Andrés Larráinzar, Chiapas (1959-2005)», en Juan Pedro Viqueira (coord.), Los indígenas de Chiapas y la rebelión zapatista. Microhistorias políticas, México, El Colegio de México, pp. 331-417.

Alpízar, Lydia y Marina Bernal, 2003, «La construcción social de las juventudes», Última Década, 19, pp. 1-20.

Bonfil Batalla, Guillermo, 1991, «Lo propio y lo ajeno: una aproximación al problema del control cultural», en Pensar nuestra cultura. Ensayos, México, Alianza, pp. 49-57.

Cancian, Frank, 1989, Economía y prestigio en una comunidad maya. El sistema religioso de cargos en Zinacantán, México, Instituto Nacional Indigenista/Secretaría de Educación Pública.

Carrasco, Pedro, 1990, «Sobre el origen histórico de la jerarquía político-religiosa de las comunidades indígenas», en Modesto Suárez (coord.), Historia, antropología y política. Homenaje a Ángel Palerm, México, Alianza, pp. 306-326.

Castro Domingo, Pablo, 1994, «El gobierno indígena en Cozoyoapan: un acercamiento político de la estructura de cargos comunitarios», en Héctor Tejera Gaona (coord.),

\footnotetext{
${ }^{33}$ Entrevista con el mayordomo mayor de San Andrés, 12 de marzo de 2014.
} 
Antropología política. Enfoques contemporáneos, México, Plaza y Valdés, pp. 503-525.

Chanse, John y William Tylor, 1985, «Associations and Charges: Historical perspective on the Mesoamerican Civil-religious Hierarchy», American Ethnologists, 12, pp. 165-184.

Costa, Pietro, 2005, Cittadinanza, Bari, Editori Laterza.

Coupland, Douglas, 1993, Generación X, Barcelona, Ediciones B.

Dehouve, Danièle, 2006, Essai sur la royauté sacrée en République mexicaine, París, CNRS.

Dumont, Louis, 1970, Homo hierarquicus. Ensayo en el sistema de castas, Madrid, Aguilar.

Erikson, Erik, 1951, Childhood and society, Nueva York, Norton.

Feixa, Carles, 1995, El reloj de arena, México, Causa joven.

Ferraroti, Franco, 1981, "Consideraciones generales de la juventud como problema social». CREA-Seminario Internacional de Investigación sobre Problemas de la Juventud. México: Memoria.

Frazer, James George, 1961, La rama dorada, México, FCE.

González de la Fuente, Ïñigo, 2011, "Comunidad, sistema de cargos y proyecto social. Una propuesta analítica de sociedades locales en México», AIBR, Revista de Antropología Iberoamericana, 6(1), pp. 83-107.

Gorza, Piero, 2006, Habitar el tiempo en San Andrés Larráinzar. Paisajes indígenas de los Altos de Chiapas, México, UNAM / El Colegio de Michoacán.

Guiteras Holmes, Calixta, 1961, Los peligros del alma, México, FCE.

Harris, Marvin, 1964, Pattern of race in the Americas, New York, Walker and Company. Hocart, Arthur Maurice, 1936, Kings and Councellors, Le Caire, Printing Office Paul Babey. Holland, William, 1963, Medicina Maya en los Altos de Chiapas, México, INI.

Huanacuni Mamani, Fernando, 2010, Vivir bien/Buen vivir. Filosofía, políticas, estrategias y experiencias regionales, Quito, Coordinadora Andina de Organizaciones Indígenas (CAOI).

Inglehart, Ronald, 1990, Culture Shift in Advanced Industrial Society, Princeton, Princeton University Press.

Korsbaek, Leif, 1992, El sistema de cargos en la antropología chiapaneca, Tuxtla Gutiérrez, Gobierno del Estado de Chiapas e Instituto Chiapaneco de Cultura.

, 2009, «El sistema de cargos, la etnografía y las comunidades indígenas», en Leif Korsbaek y Fernando Cámara Barbachano (eds.), Etnografía del sistema de cargos en comunidades indígenas del Estado de México, México, MC Editores, pp. 27-65.

Lidz, Theodore, 1973, La persona, Barcelona, Herder.

Ochiai, Kazuyasu, 1985, Cuando los santos vienen marchando, San Cristóbal de Las Casas, UNACH.

Ortelli, Paola, 2011, Relatoría del foro «Sloil slekilal kuxlejal xchi'uk mol me-eletik, xchi'uk ach' chi'eletik». Traducción de Juana Pérez de la Torre.

2012, «Aprender de los mayores: un intercambio generacional en los Altos de Chiapas», Chasqui, Revista latinoamericana de comunicación, 120, pp. 41-45.

Paoli, Antonio, 2003, Educación, autonomía y lekil kuxlejal: aproximaciones sociolingüísticas a la sabiduría de los tzeltales, México, UAM-Xochimilco. 
Pérez Ruiz, Maya Lorena (coord.), 2008, Jóvenes indígenas y globalización en América Latina, México, INAH.

Piedrasanta, Ruth, 2003, L'espace chez les Chuj de San Mateo Ixtatán (Guatemala). Représentations et dynamique historique: unité et rupture (1880-2000), tesis de doctorado inédita, Universidad Paris X Nanterre.

Pozas Arciniega, Ricardo, 1977, Chamula, México, INI.

Rodríguez, Ernesto, 1995a, «Políticas integrales de juventud: propuestas para el período 1995-1999». Montevideo, Ministerio de Educación y Cultura, Instituto Nacional de la Juventud.

, 1995b, «Políticas de juventud en América Latina en la antesala del año 2000: logros, desafíos y oportunidades», Resumen preliminar del informe final del proyecto de investigación y desarrollo «Políticas de juventud en América Latina: diseño y evaluación». Versión preparada para el Segundo Encuentro Latinoamericano de Expertos en Juventud (Santa Cruz de la Sierra, 27 al 30 de noviembre de 1995).

Rus, Jan y Robert Wasserstrom, 1980, "Civil-Religious Hierarchies in Central Chiapas: A Critical Perspective», American Ethnologist, 7(3), pp. 466-478.

Santos, Boaventura de Sousa, 2014, «¿La revolución ciudadana tiene quien la defienda?», Blog del autor, http://blogs.publico.es/espejos-extranos/2014/05/09/ [consulta: 01 de diciembre de 2014].

Turaine, Alaine, 1988, Return of the actor: Social Theory in Postindustrial Society, U.S.A., University of Minnesota Press.

Wolf, Eric, 1957, "Closed corporate peasant communities in Mesoamerica and Central Java», Southwestern Journal of Anthropology, 13(1), pp. 1-18.

Fecha de recepción: 9 de junio de 2014 Fecha de aceptación: 13 de marzo de 2015 